\title{
Q-switched fiber laser with a tungsten disulfide saturable absorber prepared by the drop casting method
}

\author{
N.N. Razak ${ }^{1}$, M. Yasin ${ }^{* 2}$, Z. Zakaria ${ }^{1}$, A.A. Latiff ${ }^{1}$ and S.W. Harun ${ }^{3}$ \\ ${ }^{1}$ Faculty of Electronic and Computer Engineering, Universiti Teknikal Malaysia Melaka, 76100 Melaka, Malaysia \\ ${ }^{2}$ Department of Physics, Faculty of Science and Technology, Airlangga University, Surabaya, Indonesia \\ ${ }^{3}$ Photonics Engineering Laboratory, Department of Electrical Engineering, University of Malaya, 50603 Kuala \\ Lumpur, Malaysia
}

Received July 15, 2017; accepted September 29, 2017; published September 30, 2017

\begin{abstract}
We experimentally demonstrate a passively Q-switched erbium-doped fiber laser (EDFL) operation by using a saturable absorber (SA) based on tungsten disulfide $\left(\mathrm{WS}_{2}\right)$. By depositing a $\mathrm{WS}_{2}$ thin film layer at the end of an optical fiber ferrule, we fabricated an SA device. The SA is incorporated into an Erbium-doped fiber laser (EDFL) cavity to generate a Q-switching pulses train operating at $1559.8 \mathrm{~nm}$. As a result, stable passively Q-switched EDFL pulses with a maximum output pulse energy of $123.2 \mathrm{~nJ}$, repetition rate of $104.1 \mathrm{kHz}$, and pulse width of $9.61 \mu$ s are achieved when the input pump power is $142.1 \mathrm{~mW}$ at a wavelength of $980 \mathrm{~nm}$.
\end{abstract}

To date, besides traditional semiconductor saturable absorber mirrors (SESAMs) [1], there are several new materials, such as carbon nanotubes (CNTs) [2], Graphene [3-4], topological isolators (TIs) [5-6], transition metal dichalcogenides (TMDs) [7] and black phosphorus [8] that have been used as passive saturable absorbers (SAs). Compared with SESAMs, these emerging materials possess the excellent virtues of a broader operation wavelength band, more excellent nonlinear optical response, and easier fabrication. Among them, a group of semiconducting TMDs, like tungsten disulfide $\left(\mathrm{WS}_{2}\right)$ and molybdenum disulfide $\left(\mathrm{MoS}_{2}\right)$, have stood out for their typical graphene-like two-dimensional (2D) layered structure, unique thickness-dependent bandgap and stronger light-matter interaction than graphene [9].

Most of the reported Q-switched lasers have low output power. For instance, Wu et al. demonstrated a Q-switched EDFL using $\mathrm{WS}_{2}$ nanosheets, which were prepared using a liquid-phase exfoliation method and embedded in a polyvinyl alcohol (PVA) film [10]. Through a mechanical exfoliation technique, the $\mathrm{WS}_{2}$ also can work as SA for Qswitching generation [11]. However, the signal-to-noise ratio of the formatted pulse is only up to $43 \mathrm{~dB}$. Chen et al. demonstrated high damage $\mathrm{WS}_{2} \mathrm{SA}$ and obtained pulse energy reaching $68.5 \mathrm{~nJ}$ [12]. In another work, the $\mathrm{WS}_{2}$ based Q-switched EDFL was demonstrated to generate a cylindrical-vector beam [13]. By combining $\mathrm{WS}_{2}$ and

*E-mail: yasin@fst.unair.ac.id
SESAM, Chen et al. successfully generated a modelocked pulse laser at the C-band region [14]. In this letter, we demonstrate a Q-switched EDFL using $\mathrm{WS}_{2}$ based SA, which was prepared using a drop casting method. Compared to the previously reported, this paper has successfully demonstrated a higher repetition rate and short pulse width size [15]. Moreover, the proposed laser produced a higher maximum pulse energy of $123 \mathrm{~nJ}$. The $\mathrm{SA}$ is developed by repeatedly dropping the $\mathrm{WS}_{2}$ onto a fiber ferrule and a dried SA device is incorporated in the ring laser cavity. This technique offers simplicity in fabrication compared to composite film, besides it allows control over the insertion loss.

In the experiment, we used a $\mathrm{WS}_{2}$ pristine flakes solution, which was obtained by a liquid exfoliation method. The $\mathrm{WS}_{2}$ flakes with the purity of more than $99 \%$ were dispersed by using a mixture of ethanol and water as a solvent. Then the dispersed solution was treated for $120 \mathrm{~min}$ by a high-power ultrasonic cleaner. After sonication, the dispersion was allowed to settle for several hours. To remove large agglomeration, the dispersion was centrifuged at $3000 \mathrm{rpm}$ for $30 \mathrm{~min}$, after which the upper supernatant was collected. The concentration of $\mathrm{WS}_{2}$ nanosheets in the dispersed solution was about $26 \mathrm{mg} / \mathrm{l}$. The $\mathrm{WS}_{2}$ solution was repeatedly dropped and dried onto a fiber ferrule until it formed a $\mathrm{WS}_{2}$ thin film layer that was strong enough to act as an SA for the laser cavity.

The deposited $\mathrm{WS}_{2}$ thin film is characterized by Raman spectroscopy as depicted in Fig. 1(a). It indicates characteristic bands at 350.8 and $420.7 \mathrm{~cm}^{-1}$, which are assigned to the in-plane $\left(\mathrm{E}_{2 \mathrm{~g}}\right)$ and out-of-plane $\left(\mathrm{A}_{1 \mathrm{~g}}\right)$ vibrational modes of $\mathrm{WS}_{2}$. Figure 1(b) shows a scanning electron microscopy (SEM) image of the $\mathrm{WS}_{2}$ thin film, which has a lateral size in the range of 50 to $150 \mathrm{~nm}$.

The fabricated SA device was integrated into an EDFL cavity as shown in Fig. 2. The cavity uses a 3-m-long erbium-doped fiber (EDF), which was pumped by a $980-$ $\mathrm{nm}$ pump via a 980/1550nm wavelength division multiplexer (WDM). The EDF used has a numerical aperture (NA) of 0.16 and erbium ion absorption of 
$23 \mathrm{~dB} / \mathrm{m}$ at $980 \mathrm{~nm}$ with core and cladding diameters of $4 \mu \mathrm{m}$ and $125 \mu \mathrm{m}$, respectively. To ensure unidirectional operation of the laser, an isolator was used. The laser signal was coupled out using a 80:20 output coupler while keeping $80 \%$ of the light to oscillate in the ring cavity. The spectral characteristic was measured using an optical spectrum analyser (OSA) with a spectral resolution of $0.02 \mathrm{~nm}$ while the temporal characteristics were measured using a $500 \mathrm{MHz}$ oscilloscope and a $7.8 \mathrm{GHz}$ radiofrequency (RF) spectrum analyser via a $1.2 \mathrm{GHz}$ photodetector.

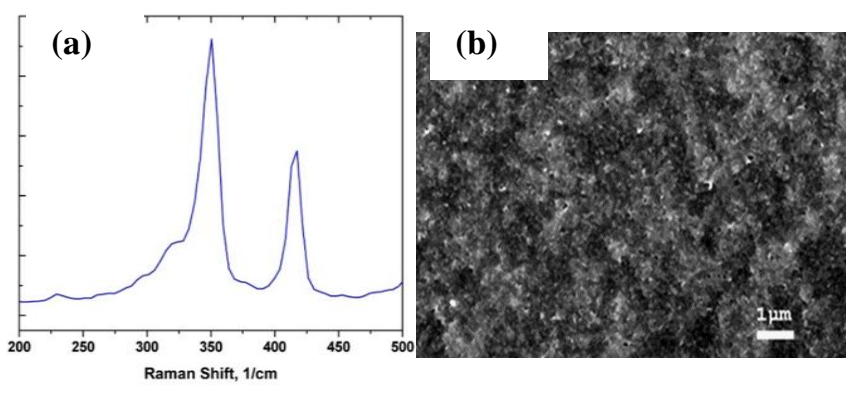

Fig. 1. Characteristics of $\mathrm{WS}_{2}$ thin film (a) Raman spectrum, (b) SEM image.

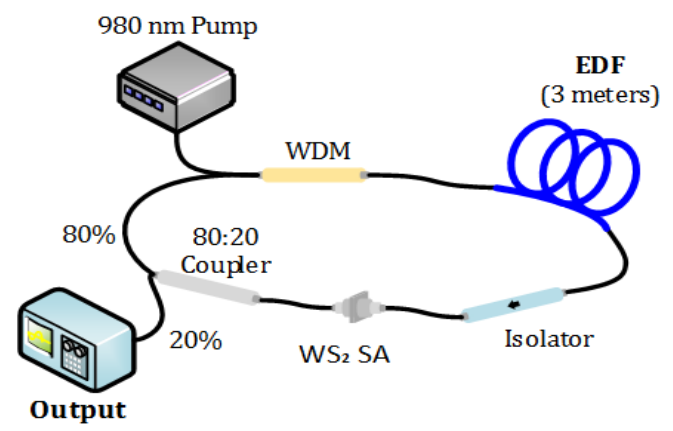

Fig. 2. Configuration of the $\mathrm{WS}_{2}$ based Q-switched EDFL.

In this experiment, a stable Q-switched pulse train is obtained at a pump power range from $60.6 \mathrm{~mW}$ to $142.1 \mathrm{~mW}$. Figure 3(a) presents the output spectrum of the Q-switched EDFL, which indicates the laser operation at $1559.8 \mathrm{~nm}$. Figure 3(b) shows a typical oscilloscope trace at a pump power of $142.1 \mathrm{~mW}$, which indicates the repetition rate of $104.1 \mathrm{kHz}$. It is also observed that the Qswitched pulse output is stable and no amplitude modulations in the pulse train can be observed, which indicates that there is no self-mode locking effect during the Q-switching operation. Moreover, the peak amplitude of the pulse is observed to be constant throughout the projection, which shows that the Q-switched pulse is stable at laboratory environment.

To verify the effects of the $\mathrm{WS}_{2}$-based SA on Qswitching, two optical fiber connectors connected by a flange are inserted in the EDFL cavity. But there is no
$\mathrm{WS}_{2}$ flakes film deposited on the end of the optical fiber connector. And the corresponding SMFs' length is not changed. No pulsed operation was observed when the pump power was varied within a wide range. This confirmed that the above Q-switched EDFL had been induced by the $\mathrm{WS}_{2}$-based SA.

To investigate the stability of Q-switching operation, we measured the signal to noise ratio of the RF signal transformed by a photodiode and analysed the spectrum with the help of an RF spectrum analyser. Figure 3(c) shows the RF spectrum at a pump power of $142.1 \mathrm{~mW}$. As seen, the $104.1 \mathrm{kHz}$ fundamental repetition rate has a signal-to-noise ratio of more than $65 \mathrm{~dB}$. This indicates that the stability of the produced pulses are excellent. In compliance with the Fourier transform, the peak of a fundamental repetition rate gradually decreases until the $6^{\text {th }}$ harmonic. Throughout the experiment, we can confirm the absence of a mode-beating frequency.
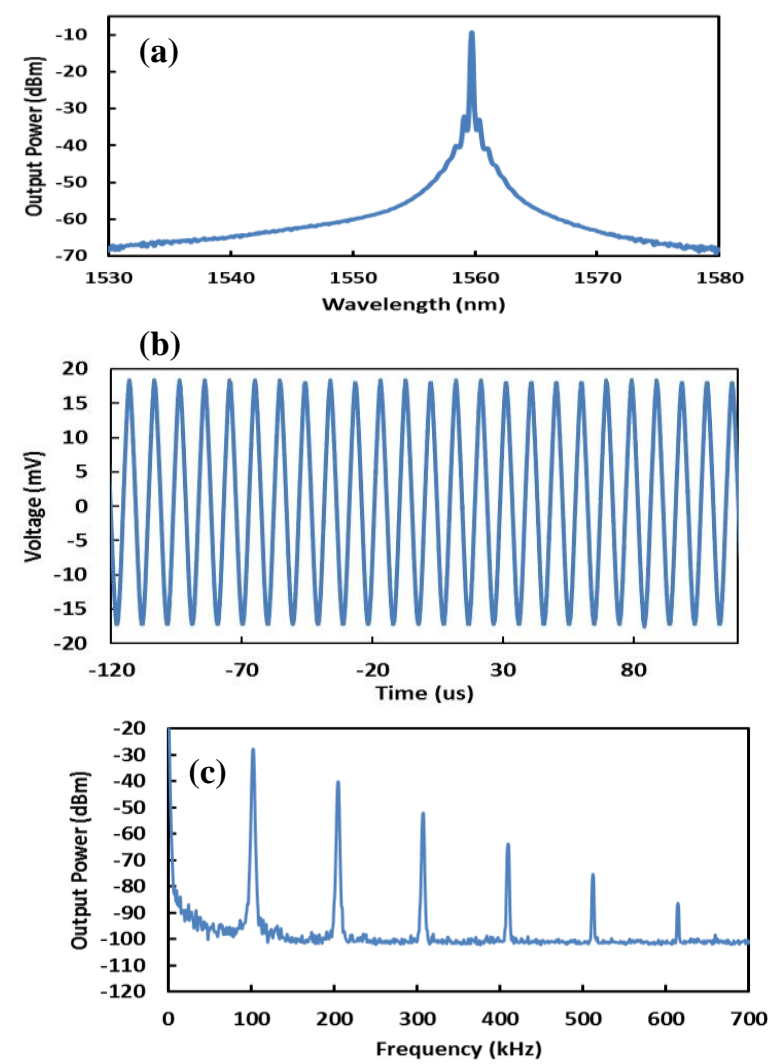

Fig. 3. Spectral and temporal characteristics at a pump power of $142.1 \mathrm{~mW}$ (a) output spectrum, (b) typical pulse train, (c) RF spectrum.

The repetition rate of output pulses monotonically increases from $70.0 \mathrm{kHz}$ to $104.1 \mathrm{kHz}$ while the pulse width reduces from $14.28 \mu \mathrm{s}$ to $9.61 \mu \mathrm{s}$, as shown in Fig. 4(a). It can be found that the repetition rate becomes higher and the pulse width becomes smaller with increasing pump power. This also presents a typical 
feature of passively Q-switched lasers. The pump rate for the upper laser level increases with increasing pump power and causes the reduction of the pulse width and the increase of the repetition rate. The demonstrated laser shows no obvious degradation under laboratory conditions for 2 hours. In our experiment, the laser begins $\mathrm{CW}$ operation when the pump power goes beyond $142.1 \mathrm{~mW}$. If the pump power is reduced to $142.1 \mathrm{~mW}$ again, Qswitching reappears. This is because $\mathrm{WS}_{2}$ exhibits saturable absorption at moderate laser intensities, while optical limiting induced by excited states absorption would occur at higher intensities.

Pulse energy is found to change with the increment of the pump power and the output power monotonically. The corresponding output power increased from $5.98 \mathrm{~mW}$ to $12.82 \mathrm{~mW}$ and the single pulse energy varied from $77 \mathrm{~nJ}$ to 123nJ, as shown in Fig. 4(b). The laser efficiency is about $9.15 \%$. The slope efficiency is reasonably high due to a relatively low cavity loss. The output pulse with an energy of $123 \mathrm{~nJ}$ was achieved at the maximum pump power of $142.1 \mathrm{~mW}$. This Q-switching performance could be further improved by optimizing the cavity design and the SA parameters such as modulation depth and insertion loss.
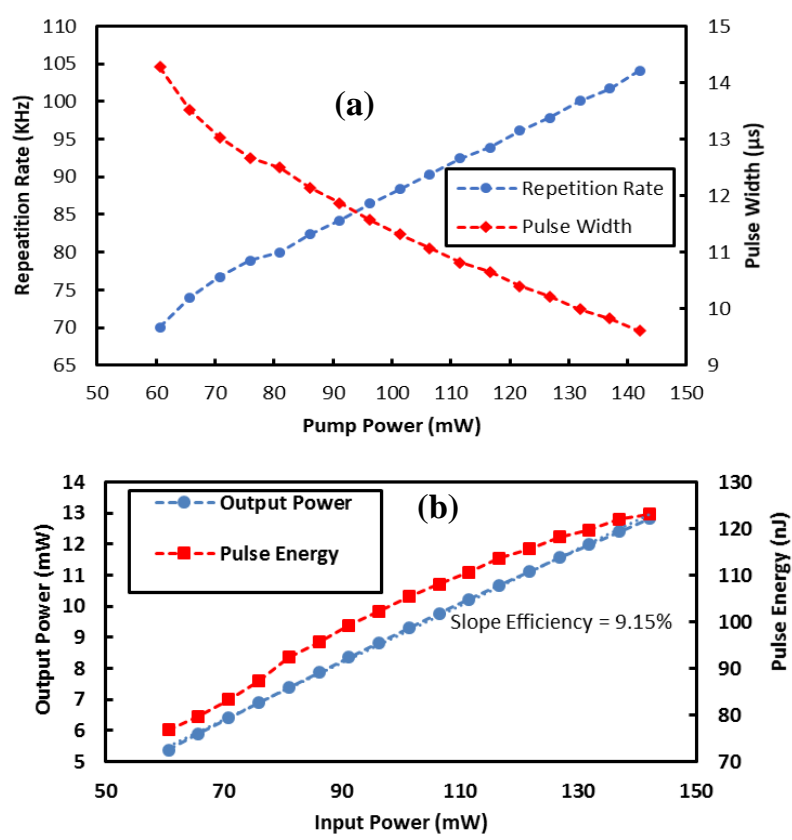

Fig. 5. Q-switching performance. (a) The pulse repetition rate and pulse width against pump power. (b) The average output power and the corresponding single-pulse energy against pump power.
In conclusion, the proposed Q-switched EDFL operating at $1559.8 \mathrm{~nm}$ using a $\mathrm{WS}_{2}$ material as an $\mathrm{SA}$ was successfully demonstrated. The SA film was fabricated by repeatedly dropping the $\mathrm{WS}_{2}$ solution onto a fiber ferrule and next the dried SA device was incorporated in the ring laser cavity. A stable Q-switched pulse train was obtained at a pump power of $60.6 \mathrm{~mW}$ and it continued to operate stably until $142.1 \mathrm{~mW}$. The corresponding pulse repetition rate was tunable from 70.0 to $104.1 \mathrm{kHz}$. The lowest pulse width was $9.16 \mu$ s and the maximum pulse energy was $123 n J$. Our experimental results suggest that $\mathrm{WS}_{2}$ is a promising material for pulsed laser applications.

\section{References}

[1] C. Gao, W. Zhao, Y. Wang, S. Zhu, G. Chen, Y. Wang, 27th International congress on High-Speed Photography and Photonics (International Society for Optics and Photonics, 2007), p. 62794G.

[2] M. Ahmed, N. Ali, Z. Salleh, A. Rahman, S. Harun, M. Manaf, et al., Opt. Laser Technol. 65, 25 (2015).

[3] M.A. Ismail, F. Ahmad, S.W. Harun, H. Arof, H. Ahmad, Laser Phys. Lett. 10, 025102 (2013).

[4] G. Sobon, J. Sotor, J. Jagiello, R. Kozinski, K. Librant, M. Zdrojek, L. Lipinska, K.M. Abramski, Appl. Phys. Lett. 101, 241106 (2012).

[5] N.H.M. Apandi, F. Ahmad, S.N.F. Zuikafly, M.H. Ibrahim, S.W. Harun, Photon. Lett. Poland 8(4), 101 (2016).

[6] J. Bogusławski, G. Soboń, K. Tarnowski, R. Zybała, K. Mars, A. Mikuła, K. M. Abramski, J. Sotor, Opt. Engin. 55, 081316 (2016).

[7] Z. Luo, Y. Huang, M. Zhong, Y. Li, J. Wu, B. Xu, H. Xu, Z. Cai, J. Peng, J. Weng, J. Lightwave Technol. 32(24), 4679 (2014).

[8] N.N. Razak, A.A. Latiff, Z. Zakaria, S.W. Harun, Photon. Lett. Poland 9(2), 72 (2017)

[9] D. Mao, Y. Wang, C. Ma, L. Han, B. Jiang, X. Gan, S. Hua, W. Zhang, T. Mei, J. Zhao, Sci. Rep. 5, 7965 (2015).

[10] K. Wu, X. Zhang, J. Wang, X. Li, J. Chen, Opt. Expr. 23, 11453 (2015).

[11] K. Lau, A. Latif, M.A. Bakar, F. Muhammad, M. Omar, M. Mahdi, Appl. Phys. B 123, 221 (2017).

[12] H. Chen, Y. Chen, J. Yin, X. Zhang, T. Guo, P. Yan, Opt. Expr. 24, 16287 (2016).

[13] J. Lin, K. Yan, Y. Zhou, L. Xu, C. Gu, Q. Zhan, Appl. Phys. Lett. 107, 191108 (2015).

[14] H. Chen, Y. Chen, J. Yin, X. Zhang, T. Guo, P. Yan, Opt. Expr. 24, 16287 (2016).

[15] K. Mohamed, B. Hamida, S. Khan, L. Hussein, M. Ahmat, E. Ismail, N. Kadir, A. Latif, S. Harun, Ukrainian J. Phys. Optics 18(1), 20(2017). 